\title{
Sulfone-Containing Methacrylate Homopolymers: Wetting and Thermal Properties
}

Shota Fujii and Thomas J. McCarthy*

Polymer Science and Engineering Department, University of Massachusetts, Amherst, Massachusetts 01003, United States

Table of Contents

NMR Spectra

GPC

XPS 
${ }^{1} \mathrm{H}-\mathrm{NMR}$ Spectrum of 1

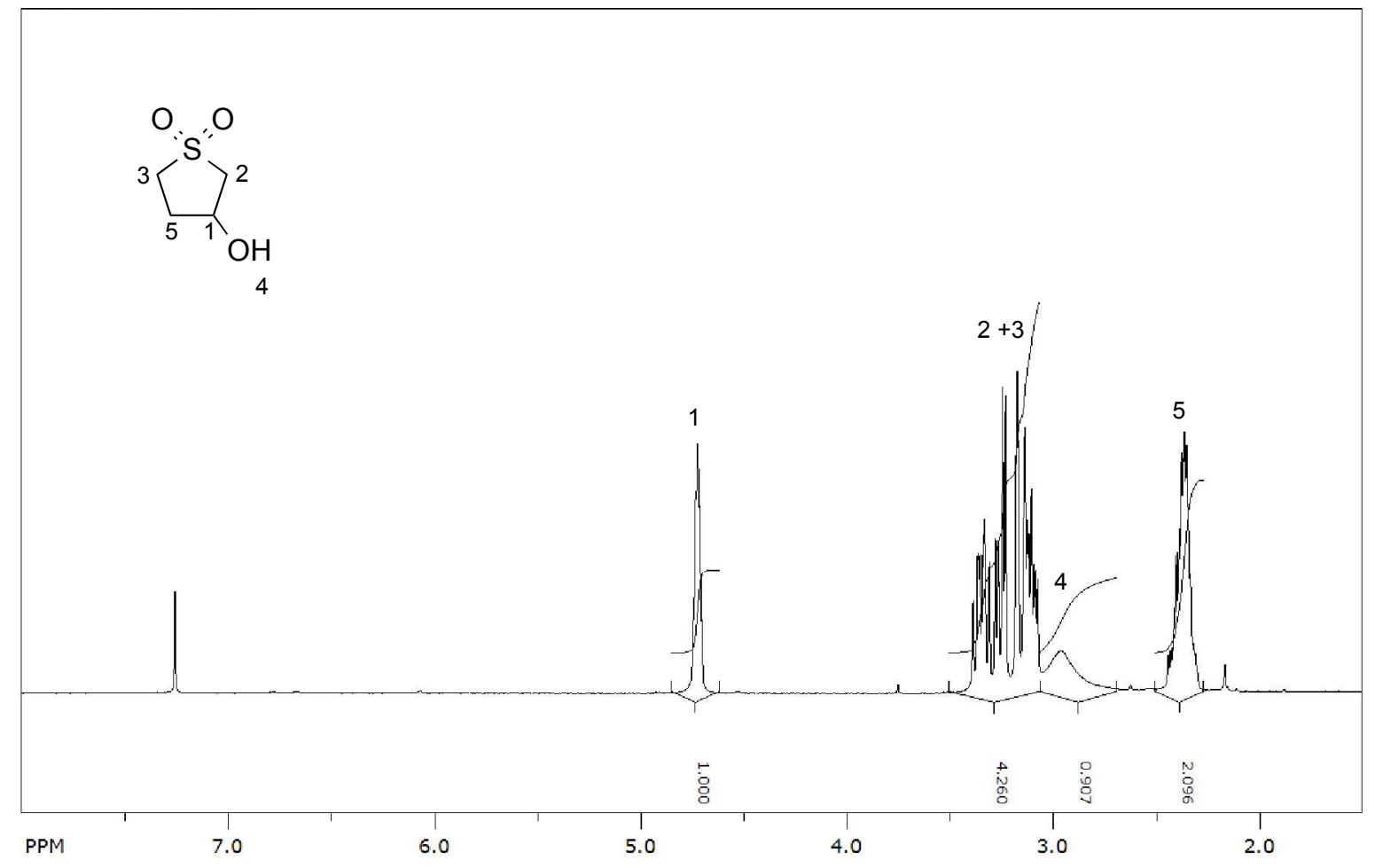

${ }^{1} \mathrm{H}$-NMR Spectrum of 2

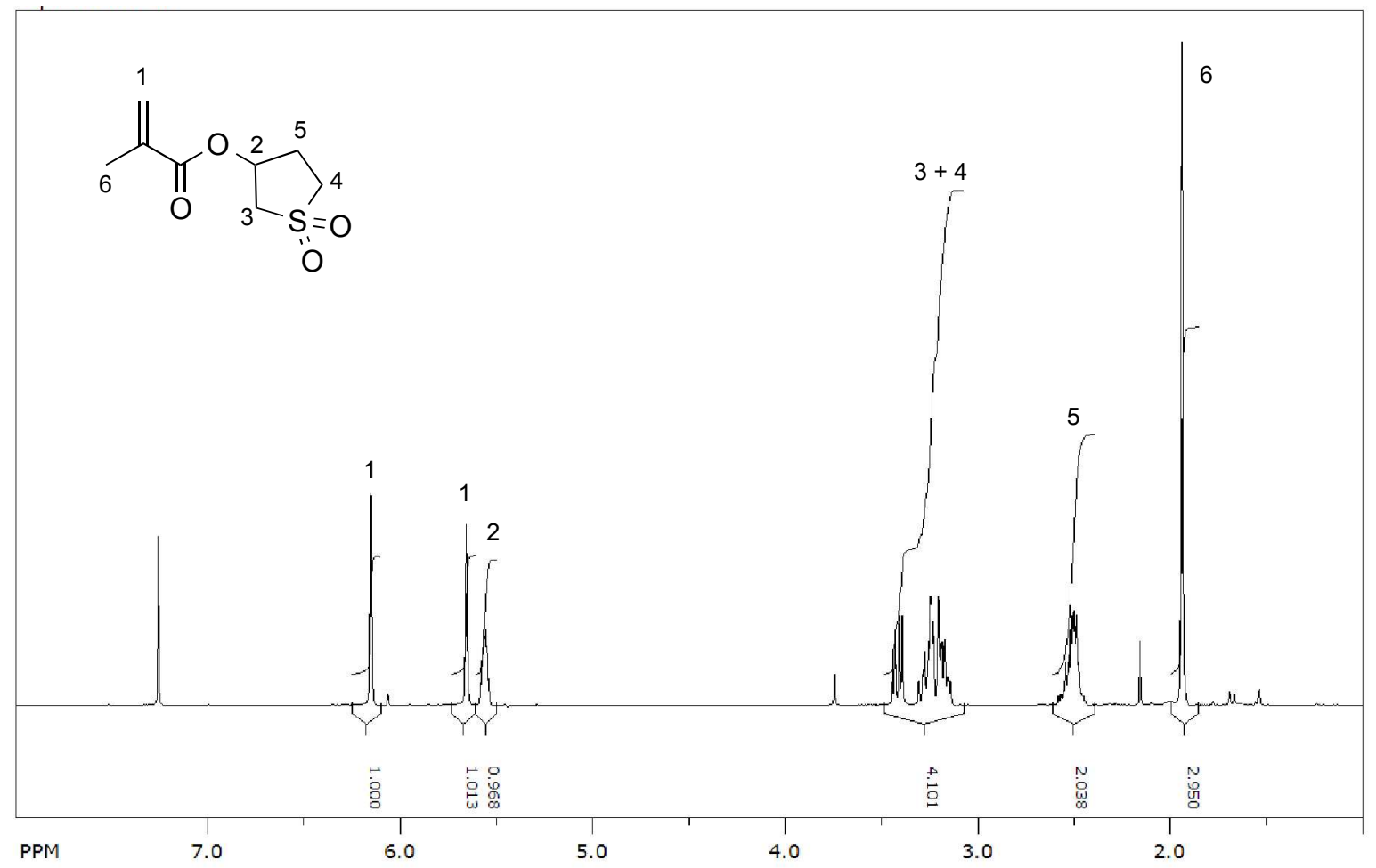


${ }^{13} \mathrm{C}-\mathrm{NMR}$ Spectrum of $\mathbf{2}$

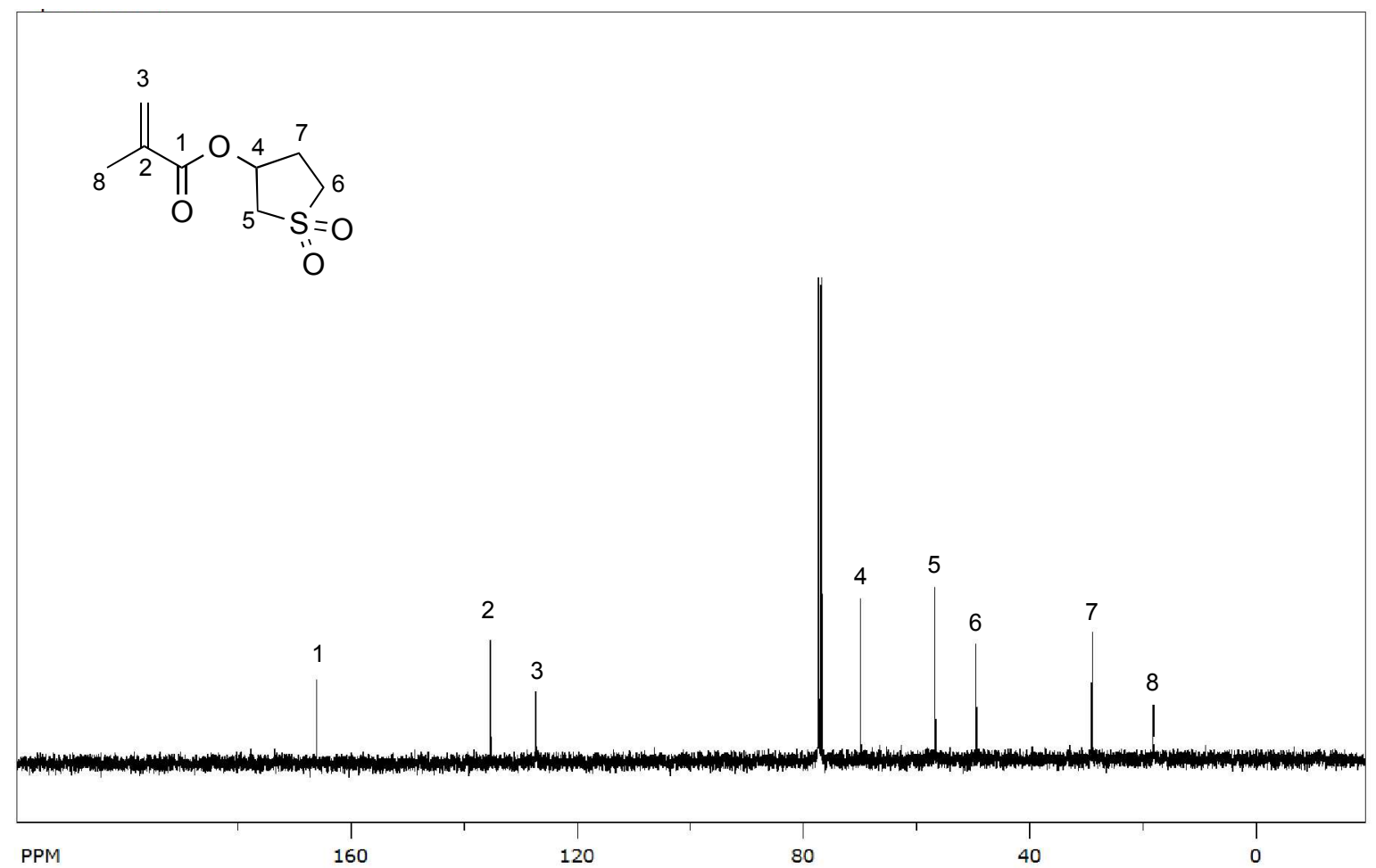

${ }^{1} \mathrm{H}-\mathrm{NMR}$ Spectrum of PSMA

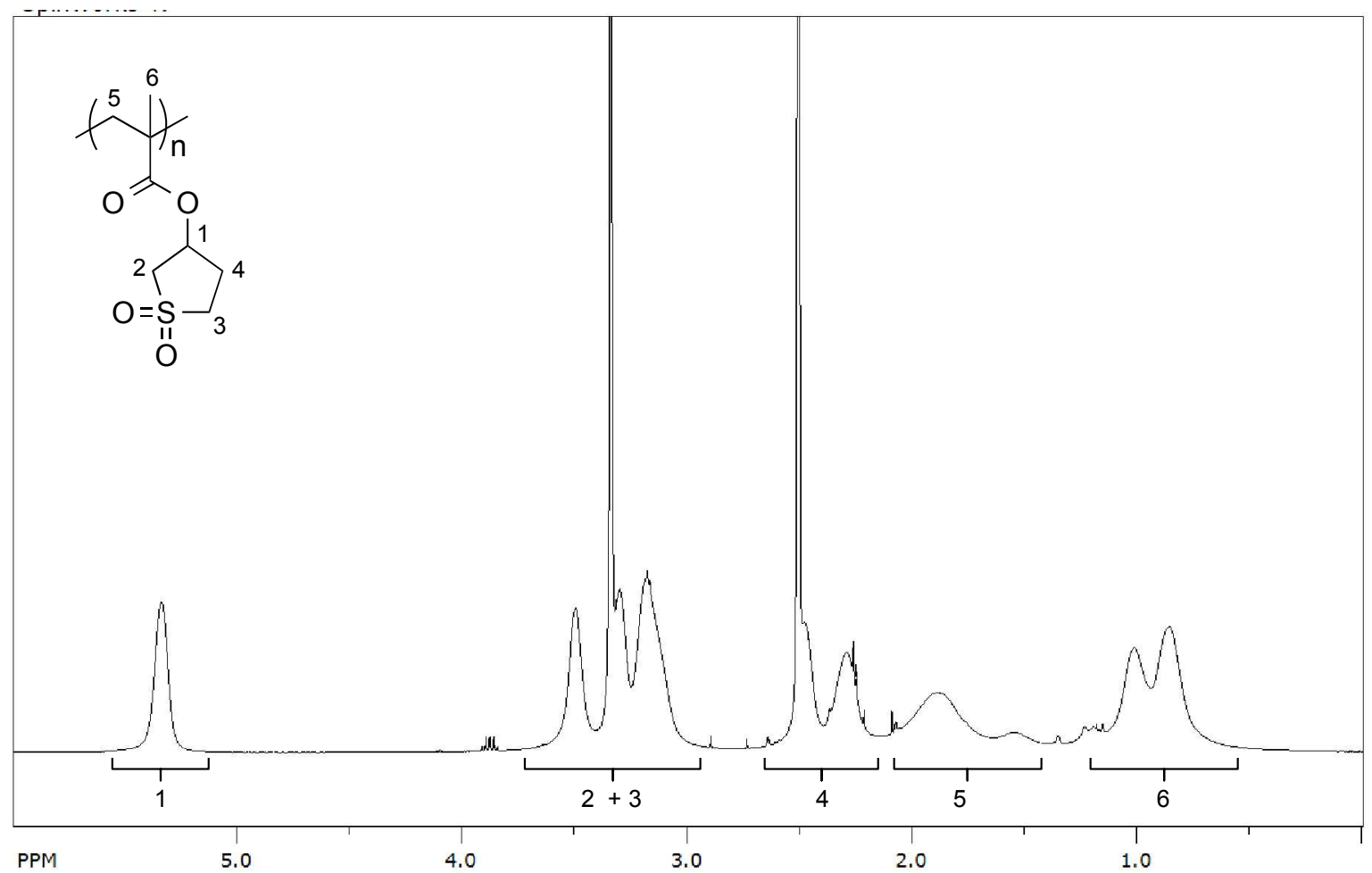


${ }^{1} \mathrm{H}-\mathrm{NMR}$ Spectrum of $\mathbf{3}$

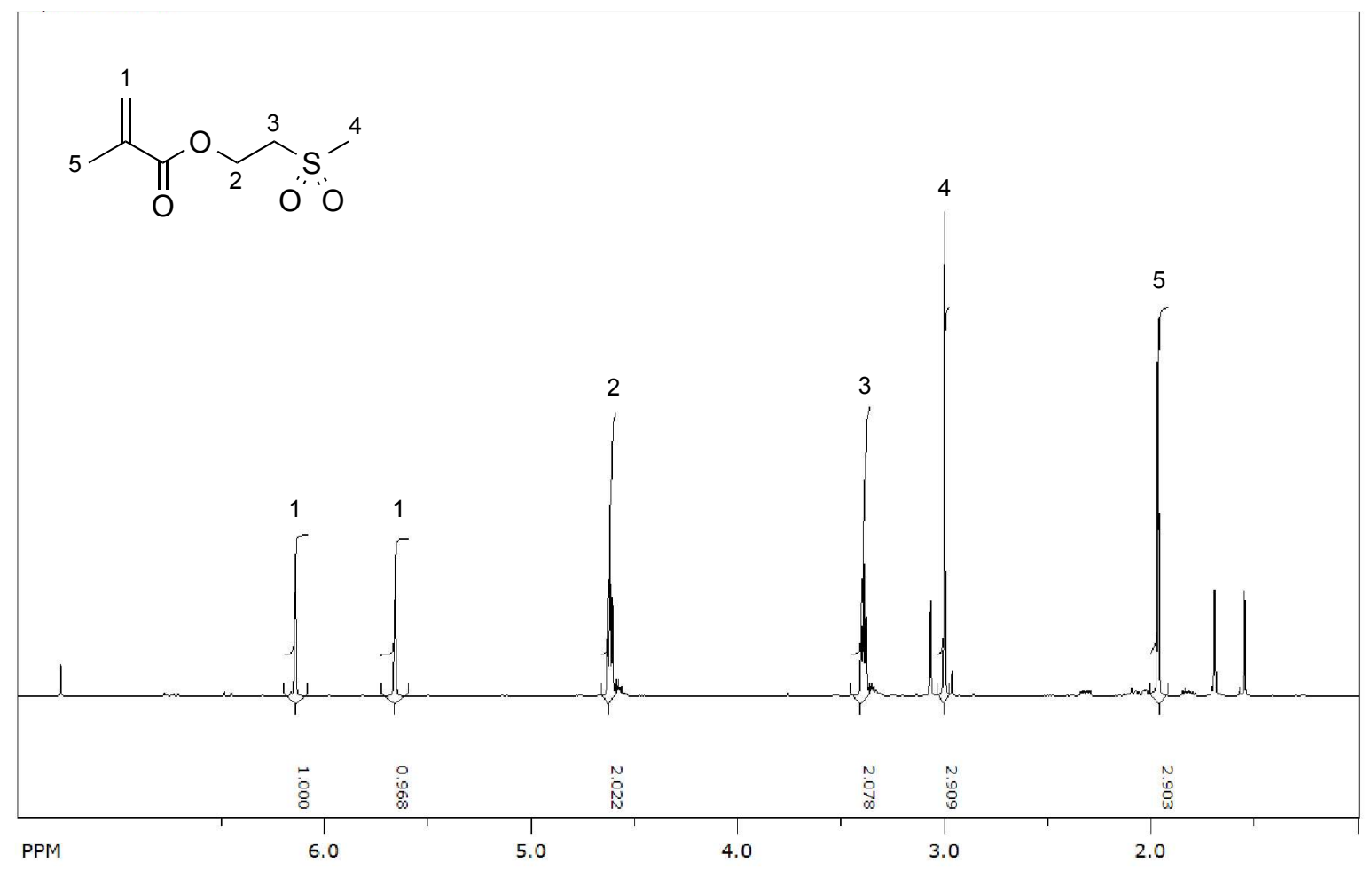

${ }^{13} \mathrm{C}-\mathrm{NMR}$ Spectrum of 3

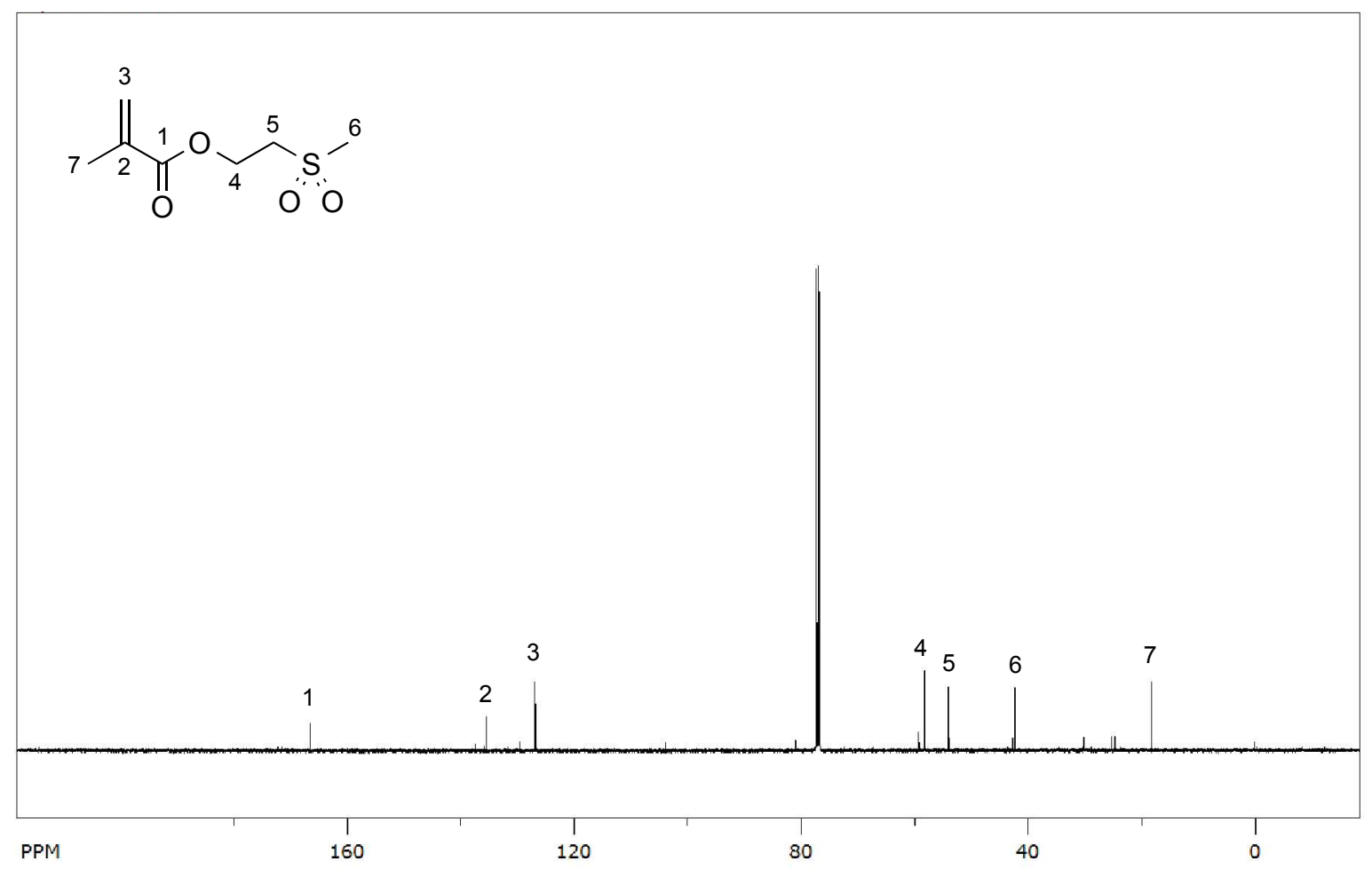


${ }^{1} \mathrm{H}-\mathrm{NMR}$ Spectrum of PMSEMA

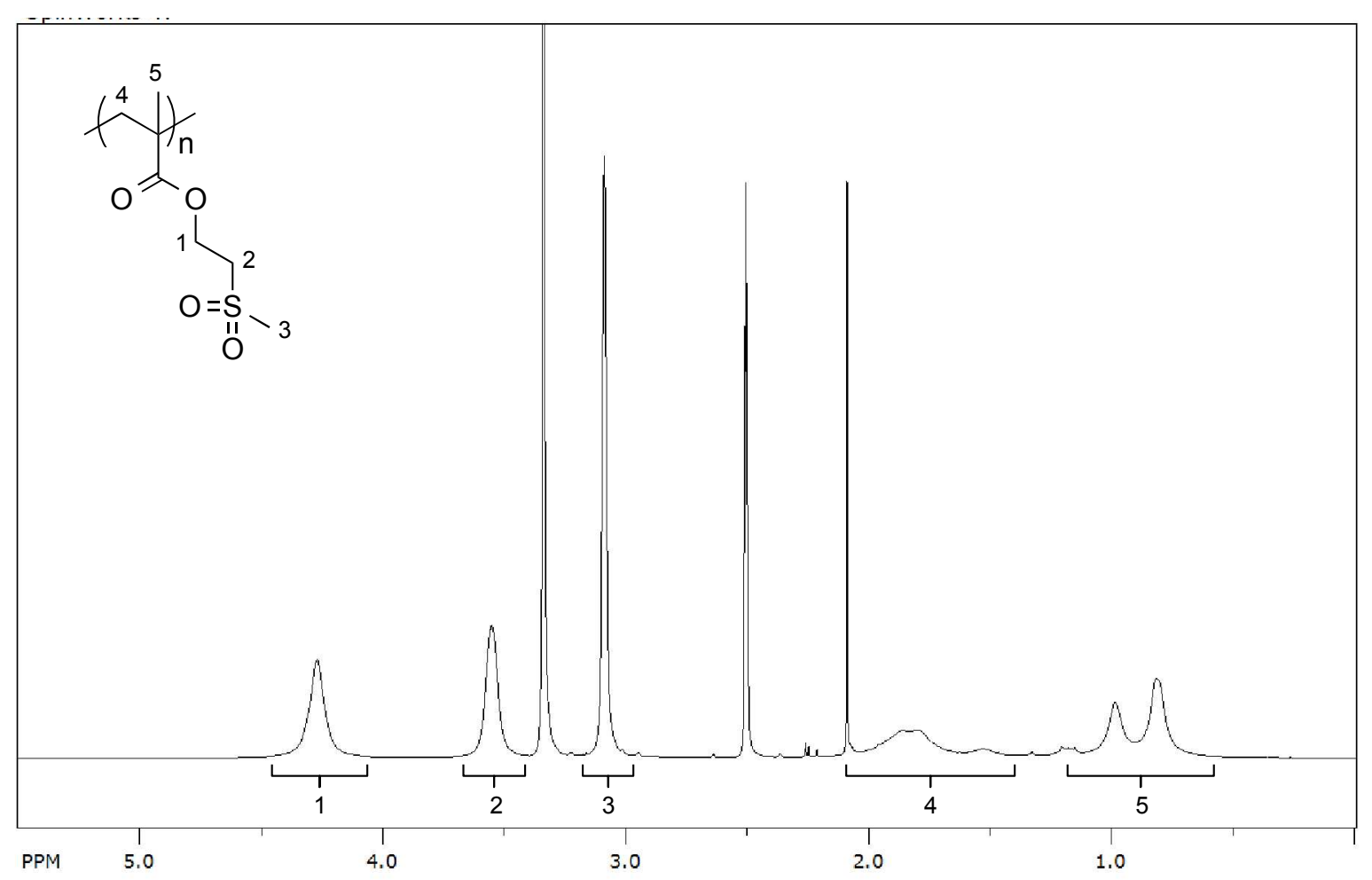

${ }^{1} \mathrm{H}-\mathrm{NMR}$ Spectrum of 4

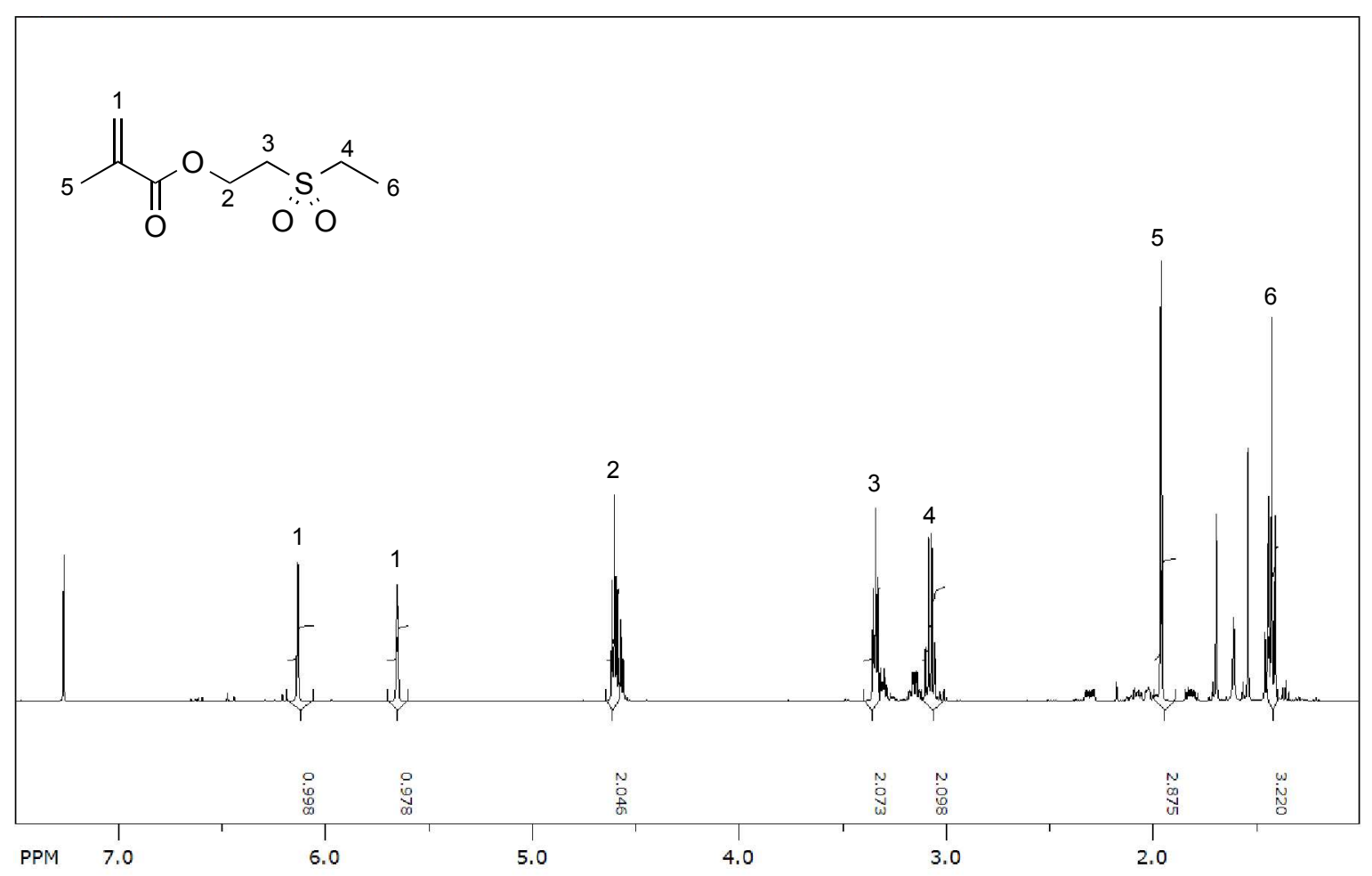


${ }^{13}$ C-NMR Spectrum of $\mathbf{4}$

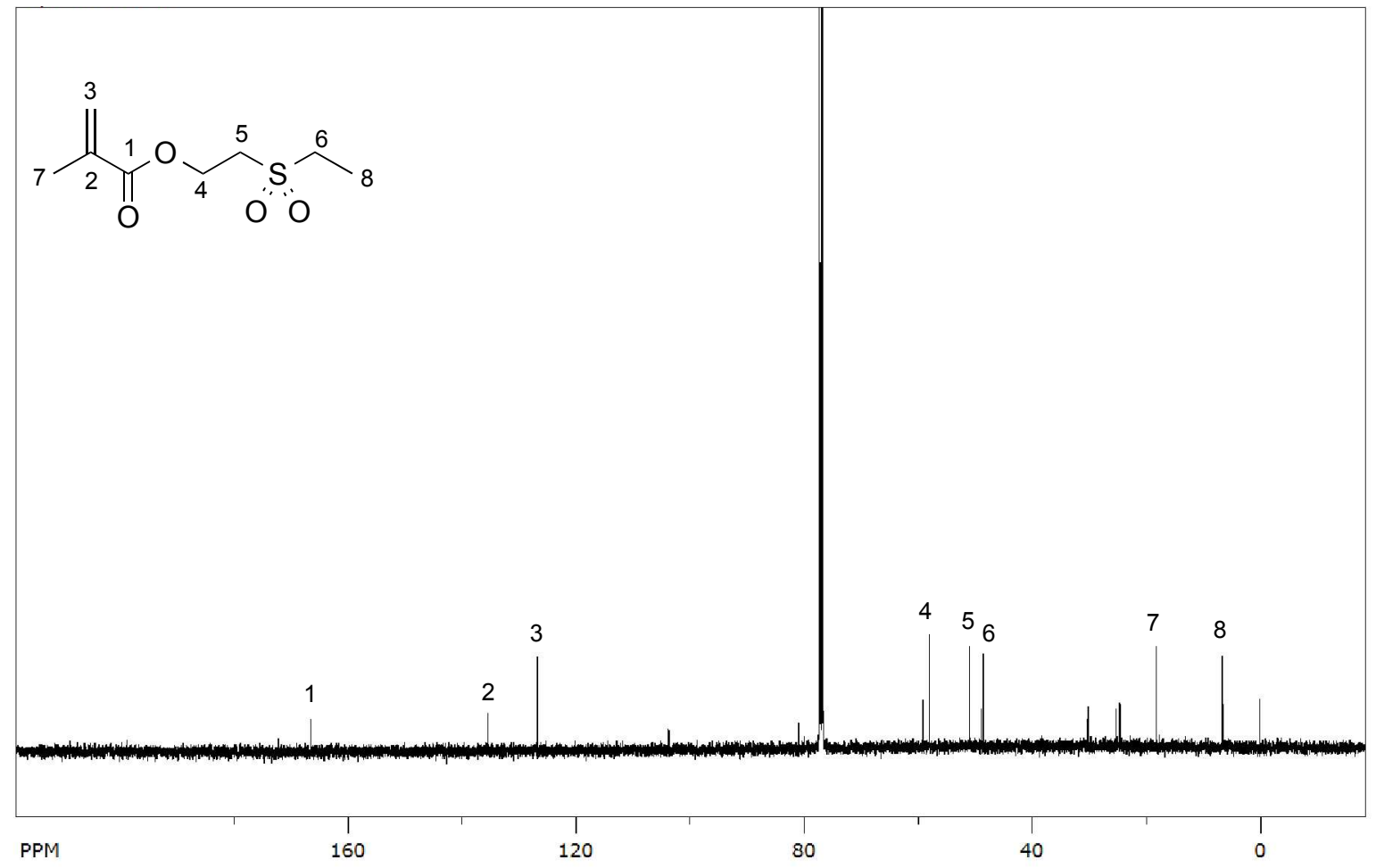

${ }^{1} \mathrm{H}-\mathrm{NMR}$ Spectrum of PESEMA

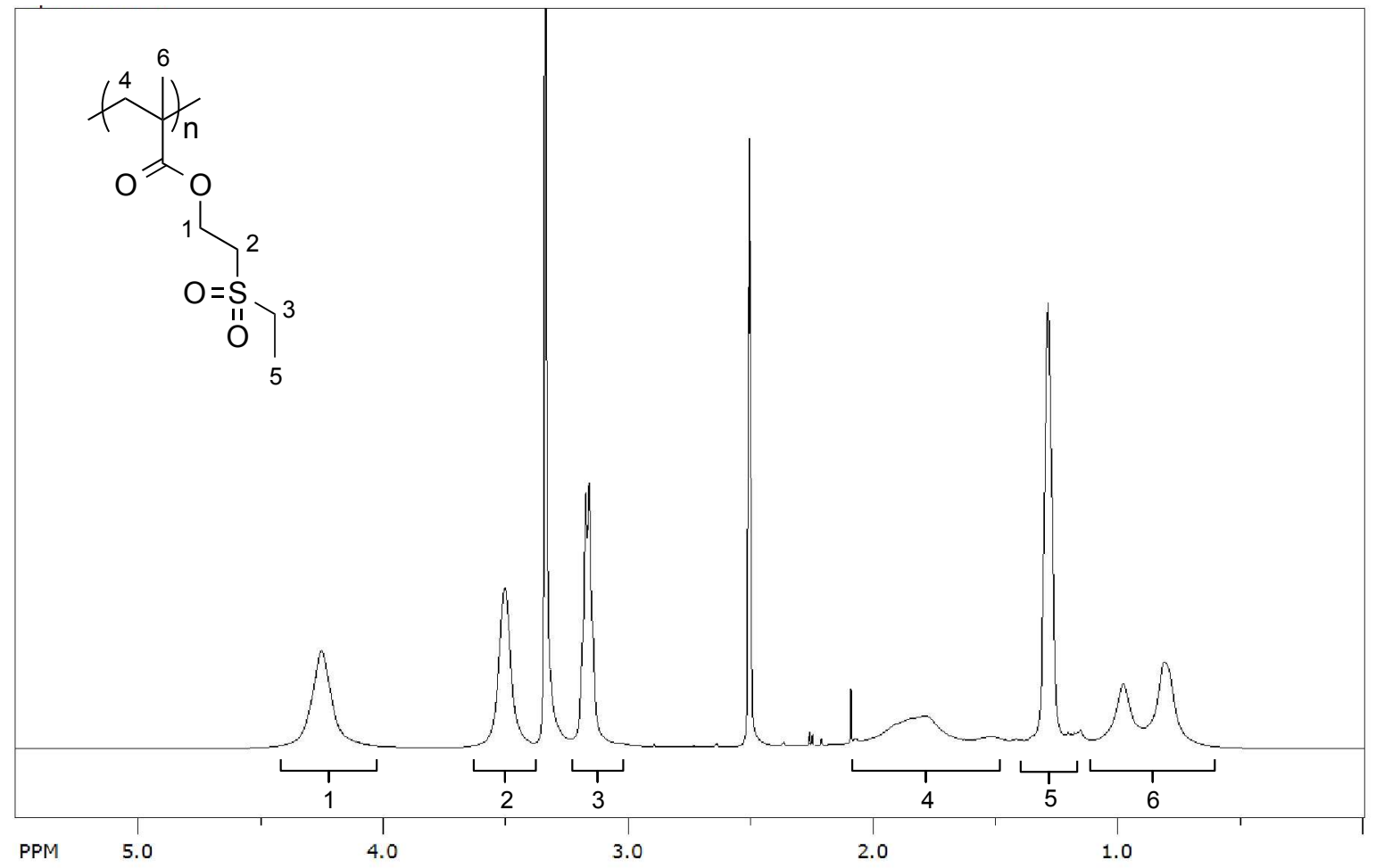


GPC

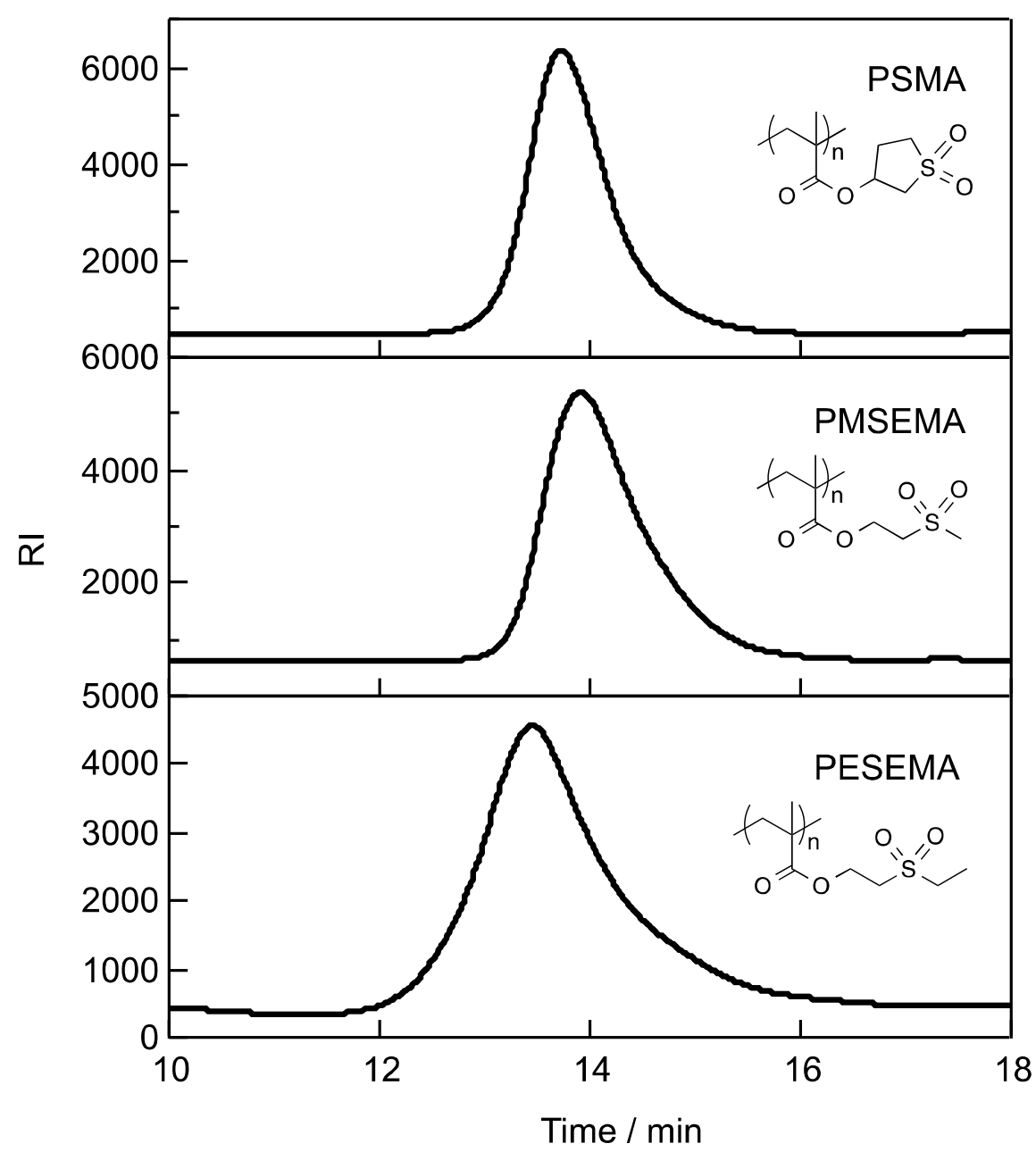

Figure S1. GPC traces for PSMA, PMSEMA and PESEMA eluted by DMF containing $0.01 \mathrm{M} \mathrm{LiCl}$ at $50 \square$. 
(a)

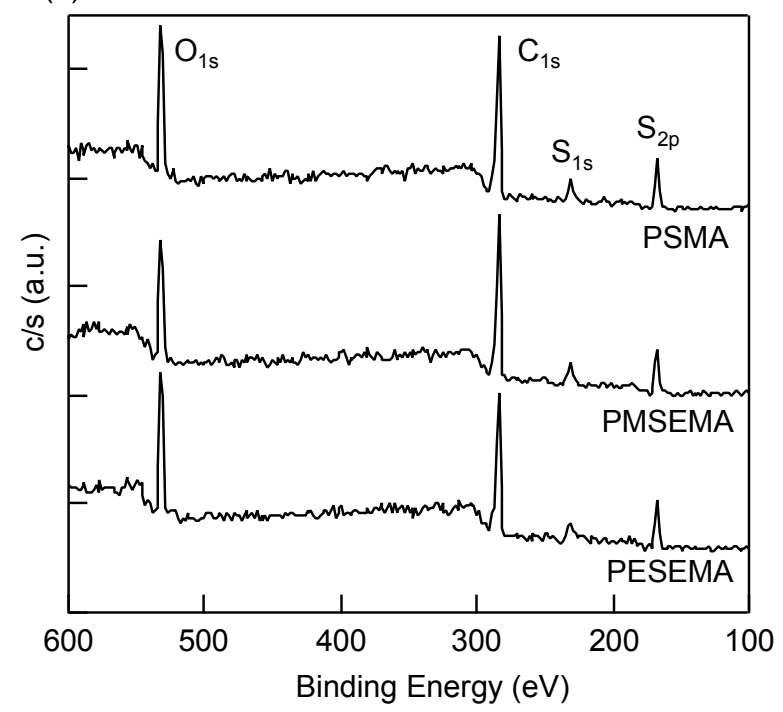

(b)

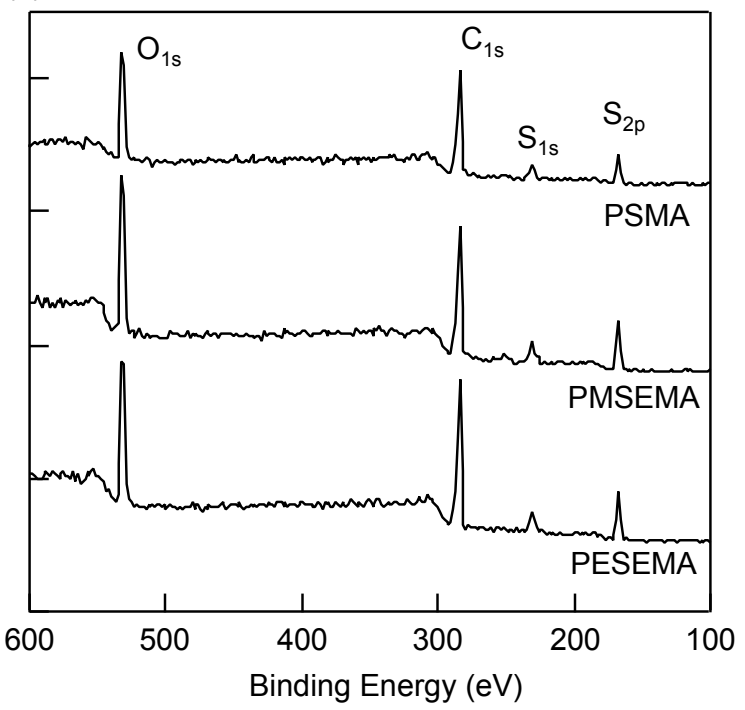

Figure S2. XPS survey spectra for sulfone polymer films at different takeoff angle: (a) $15^{\circ}$, (b) $75^{\circ}$

Table S1. XPS Analysis of Sulfone polymer films

\begin{tabular}{cccc}
\hline & \multicolumn{3}{c}{ XPS atomic $\%^{a}$} \\
\cline { 2 - 4 } sample & $\mathrm{C}$ & $\mathrm{O}$ & $\mathrm{S}$ \\
\hline \multirow{2}{*}{ PSMA } & 64.9 & 28.3 & 6.85 \\
& 63.5 & 29.8 & 6.80 \\
PMSMA & 69.3 & 24.7 & 6.10 \\
& 62.8 & 30.6 & 6.65 \\
PESMA & 65.3 & 27.8 & 6.95 \\
& 62.8 & 30.5 & 6.85 \\
\hline
\end{tabular}

${ }^{a}$ Upper rows are $15^{\circ}$ takeoff angle data and lower rows are $75^{\circ}$ data. 